\title{
Network Development in Biological Gels: Role in Lymphatic Vessel Development ${ }^{1}$
}

\author{
Tiina Roose ${ }^{\mathrm{a}, *}$, Andrew C. Fowler ${ }^{\mathrm{b}}$ \\ ${ }^{a}$ Centre for Industrial and Applied Mathematics and Centre for Mathematical Biology, \\ Mathematical Institute, 24-29 St Giles', Oxford OX1 3LB, UK \\ ${ }^{b}$ Department of Mathematics and Statistics, University of Limerick, Limerick, Republic of \\ Ireland
}

Received: 6 November 2007 / Accepted: 2 April 2008

(c) Society for Mathematical Biology 2008

\begin{abstract}
In this paper, we present a model that explains the prepatterning of lymphatic vessel morphology in collagen gels. This model is derived using the theory of two phase rubber material due to Flory and coworkers and it consists of two coupled fourth order partial differential equations describing the evolution of the collagen volume fraction, and the evolution of the proton concentration in a collagen implant; as described in experiments of Boardman and Swartz (Circ. Res. 92, 801-808, 2003). Using linear stability analysis, we find that above a critical level of proton concentration, spatial patterns form due to small perturbations in the initially uniform steady state. Using a long wavelength reduction, we can reduce the two coupled partial differential equations to one fourth order equation that is very similar to the Cahn-Hilliard equation; however, it has more complex nonlinearities and degeneracies. We present the results of numerical simulations and discuss the biological implications of our model.
\end{abstract}

Keywords Biomedical modeling $\cdot$ Mathematical biology $\cdot$ Mathematical modeling

\section{Introduction}

Even though the existence of lymphatic vessels has been known since the seventeenth century, until very recently, not very much was known about their functioning and development. This was due to a failure to understand their importance in the proper functioning of tissues. However, in last the 10 years, lymphatics have come to the forefront of biomedical research, largely due to findings highlighting their importance to cancer growth and metastasis (Stacker et al., 2002). Thus, there are now a large number of experimental studies on the molecular and micromechanical factors that control lymphatic function and development.

\footnotetext{
${ }^{*}$ Corresponding author.

E-mail address: roose@maths.ox.ac.uk (Tiina Roose).

${ }^{1}$ This work was supported by the Royal Society (London) by the award of a University Research Fellowship.
} 
The fundamental role of lymphatics is to collect excess interstitial fluids, tissue waste products, and plasma proteins from tissue and return them to the blood. Tissues require fluid and nutrients and these are supplied to them by blood vessels. After the fluid has performed its function, it is reabsorbed either by the lymphatics or by post capillary venules; the dominant contribution in most cases being due to the lymphatics. Thus, when the lymphatic system is unable to function, the interstitial pressure becomes heightened and the tissues become swollen. In addition, since reabsorption drives the flow of fluids through tissues, failure of the lymphatic system usually results in much slower, mainly diffusive, movement of nutrients into tissues, and the accumulation of waste products. In order to perform its important task, the lymphatic system has evolved into an elaborate, highly branched, highly valved, drainage network. In addition to its primary task, the lymphatic system also plays an important part in the immune system. In particular, lymphocytes, which reside and multiply in the lymphatic system, clean lymph fluid of bacteria and other contaminants. Thus, diseases of the lymphatic system often result in compromised immune competence.

Many medical conditions have now been linked to a malfunctioning of the lymphatic system, for example lymphedema, Melkersson-Rosenthal-Meischer syndrome, Kaposi sarcoma, and lymphatic filariasis. Lymphatic filariasis is a parasitic disease that is thought to be globally the second leading cause of permanent and long-term disability (Jussila and Alitalo, 2002). In recent years, the lack of lymphatic function in solid tumors has been identified as one cause for hindered delivery of chemotherapeutic drugs to solid tumors (Jain, 2001). Tumor metastasis is also thought to involve lymphatics as one, if not the primary, pathway (Cassella and Skobe, 2002; Skobe et al., 2001; Stacker et al., 2002).

In this paper, we will derive a model that sheds some light on the aspects that control lymphatic vessel development, i.e., lymphangiogenesis. In particular, we concentrate our efforts on trying to explain the experimental findings of Boardman and Swartz (2003). Boardman and Swartz found that the development of the lymphatic network within a collagen implant in a mouse tail is preceded by the development of a fluid flow network within it. Thus, it appears that collagen prepatterning plays a role in guiding the lymphatic development. In particular, it precedes the migration of the lymphatic endothelial cells.

Although there are several authors who have noted the existence of preferential fluid flow channels and networks in the tissue and calculated their transport properties (Landis and Pappenheimer, 1963; Levick, 1987; Watson et al., 1980; Watson and Grodins, 1978) to our knowledge there are no detailed mathematical models describing the development of this network in biogels or living tissues. Cogan and Keener (2005) develop a model for the development of a single channel between two constraining boundaries. However, we will show that more complex structures, which are reminiscent of a mature primary lymphatic network, could come about in a collagen gel.

In their landmark experiments, Boardman and Swartz (2003) cut away a small thin circular layer of skin around the tail of a mouse where the lymphatics reside leaving the vascular structure of the mouse tail intact. They then filled this area with $3 \%$ collagen and saw that before the lymphatic endothelial cells migrated into this collagen implant spontaneous fluid flow network had formed within the implant. Although the experiments need to be refined, using higher spatial and temporal resolution, we hypothesize that these channels were formed due to the intricate interplay between the collagen gel and the solutes (in particular protons) that infiltrated the gel after it was transplanted around the mouse tail. Collagen is a fibrous protein that makes up most of the connective tissue 


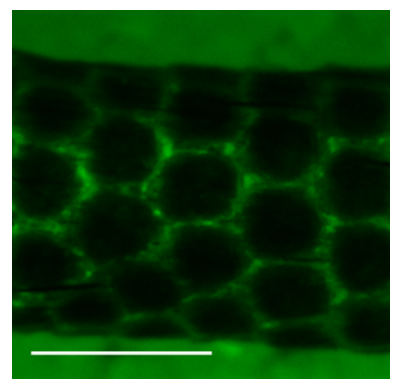

Fig. 1 Figure showing the visualization of lympatic network in the mouse tail. Green color shows the location of lymphatic channels. White bar corresponds to $1 \mathrm{~mm}$. Figure generously provided by Melody Swartz and Joseph Rutkowski from École Polytechnique Fédérale de Lausanne. (Colour figure online.)

of animals (Vogel, 2003). It consists of long thin fibres that are bound together to give strength to the tissue. In between the fibres, one finds an interstitial fluid. Crucially, it has been found that collagen gels, and hence also biological tissues, can swell and be broken down by acidic pH (Nussbaum, 1986; Nussbaum and Grodzinsky, 1981). We will model the interaction between the elastic/rubber like properties of collagen and protons with the aim of explaining how this can lead to development of a fluid flow network with a morphology that is similar to the one found in experiments of Boardman and Swartz (2003) (see also Fig. 1).

In Section 2, we will derive a model for the collagen gel and proton interaction based on the theory by Flory for long chain polymeric mixtures by considering the free energy of the mixture. This results in two coupled fourth order equations for the collagen volume fraction and the proton concentration. In Section 3, we will present the linear stability analysis of the model and show that for proton concentration above a certain level, a spatially uniform steady state is unstable to small random perturbations, and thus spatial patterns will develop. In Section 4, we use the results of the linear stability analysis to guide us on reducing the order of the system by conducting a long wavelength reduction of the coupled equations that results in a single reduced equation for the collagen volume fraction. The equation that we derive is similar to the Cahn-Hilliard equation, however, it has more complicated nonlinearities and degeneracies. We solve this equation numerically using finite element simulations that have been confirmed using a spectral method. Finally, in Section 5, we discuss our results and outline the future avenues of research.

\section{Model development}

We consider the collagen implant to consist of two phases, the fibre phase $\phi$, and the fluid phase $1-\phi$. Conservation equations for these two phases can be written as

$$
\phi_{t}+\nabla \cdot\left(\phi \mathbf{v}_{p}\right)=0, \quad \text { and } \quad-\phi_{t}+\nabla \cdot\left[(1-\phi) \mathbf{v}_{f}\right]=0
$$

where $\mathbf{v}_{p}$ is the fibre velocity, $\mathbf{v}_{f}$ is the fluid velocity, and $\phi_{t}$ is shorthand for $\partial \phi / \partial t$. 
Similarly to Milner (1993), Tanaka (1997), we define the volume averaged velocity to be

$$
\mathbf{v}=\phi \mathbf{v}_{p}+(1-\phi) \mathbf{v}_{f}
$$

Therefore, it follows from the conservation equations (1) that

$$
\nabla \cdot \mathbf{v}=0
$$

We assume that the solute, i.e., protons, matrix metalloproteases, etc., is present in the fluid phase of the implant, and that this solute can be carried by the convective movement of fluid and it can also diffuse in the fluid phase. Thus, we take the equation for the solute concentration $c$ to be given by

$$
c_{t}+\nabla \cdot\left[c(1-\phi) \mathbf{v}_{f}\right]=\nabla \cdot[D(\phi) \nabla c],
$$

where $c_{t}=\partial c / \partial t$ and $D(\phi)=D_{0} e^{-a \phi^{b}}$ is the effective diffusion of solute in the fluid phase (Clague and Phillips, 1997). In this equation, we are assuming that there are no source or sink terms for solute within the sample. We believe this to be a reasonable assumption in the case of protons, for example, which are usually abundant in the fluid phase and any depletion of them due to their interaction with collagen can be considered negligible. Also, since the collagen implant is acellular, it is reasonable to assume that there are no source terms for $c$ present inside the implant.

We now need to derive expressions that describe the fibre and fluid velocities, $\mathbf{v}_{p}$ and $\mathbf{v}_{f}$, respectively. We follow the approach first outlined by Flory (1953) for rubber elasticity by assuming that collagen behaves, as a first approximation, similarly to long chain rubber molecules. For this approach, we need to describe the interaction between the collagen fibres and the fluid. After Boudaoud and Caieb (2003), Flory (1953) and others, we consider the free energy of the gel to be a sum of three terms: the fluid-collagen interaction energy $\left(F_{\text {interaction }}\right)$, the fibre network elastic energy $\left(F_{\text {elastic }}\right)$, and the correlation energy $\left(F_{\text {correlation }}\right)$. Thus, the total energy of the collagen gel is given by

$$
F=F_{\text {interaction }}+F_{\text {elastic }}+F_{\text {correlation }} .
$$

We will now discuss each of these three terms briefly.

Following Boissonade (2003), Flory (1953), Wolgemuth et al. (2004), the interaction term is given by

$$
F_{\text {interaction }}=\int_{\Omega} f_{\text {interaction }} d V
$$

where $f_{\text {intraction }}$ is the free energy density of the interaction that determines the number and stability of different possible phases. It is usually derived in statistical mechanics by considering, for example, the configurations that different Gaussian chains can take in a given solvent (Doi and Edwards, 1986; Doi, 1983). Here, we take it to be given by the Flory-Huggins expression (Flory, 1953)

$$
f_{\text {interaction }}=\frac{k_{B} T}{v}\{(1-\phi) \ln (1-\phi)+\chi \phi(1-\phi)\},
$$


where $k_{B} T$ is the thermal energy, $\chi$ is the Flory fiber-solvent interaction parameter, and $v$ is the volume of the monomer (Flory, 1953; Wolgemuth et al., 2004). The first term expresses the entropic contribution to the free energy and the second term expresses the enthalpic contribution.

Similarly, Flory (1953) derived an expression for an elastic energy that would be present in a network of cross-linked long chain molecules, i.e., $F_{\text {elastic }}=\int_{\Omega} f_{\text {elastic }} d V$, where

$$
f_{\text {elastic }}=\frac{k_{B} T}{v} \frac{1}{2 N_{x}}\left(\phi_{0}-\phi-\frac{1}{3} \phi \ln \frac{\phi_{0}}{\phi}\right),
$$

where $N_{x}$ is the number of monomers between the cross-links, and $\phi_{0}$ is the equilibrium/initial polymer volume fraction.

We take the correlation energy to be given by classical Orstein-Zernike form for free energy (Boudaoud and Caieb, 2003; Wolgemuth et al., 2004), i.e.,

$$
F_{\text {correlation }}=\int_{\Omega} \frac{k}{2}(\nabla \phi)^{2} d V,
$$

where $k$ is proportional to correlation length.

Crucially, in this paper, we will hypothesize that the distance between the cross-links $N_{x}$, and, therefore, the elastic energy of the gel is a function of chemicals in the fluid phase, i.e., we assume that the chemicals in the fluid phase can break down the cross-links and lead to swelling in the absence of other forces; an observation that is supported by the experiments of Nussbaum (1986), Nussbaum and Grodzinsky (1981) on the swelling of collagen gel due to changes in solute (for example, proton) concentration. Motivated by this, we take the average distance between the cross-links to be a function of solute concentration

$$
N_{x}=N_{x 0} e^{\alpha \frac{c-c_{0}}{c \infty-c}},
$$

where $N_{x 0}$ is the average equilibrium distance between the cross-links at the reference state $c_{0}$, and $c_{\infty}$ is the concentration of solute in the fluid phase when the cross-links have completely disappeared, i.e., when $N_{x} \rightarrow \infty$. This is, of course, a phenomenological function and the choice of it was determined by the qualitative behavior observed in the cited experiments. In the absence of any detailed experimental evidence, we are not going to worry about the exact functional form of $N_{x}$. Instead, we will be interested only in the early stages of patterning and, therefore, in the linear stability analysis shown later only, the value of $d N_{x} / d c$, evaluated at the reference state, influences the onset of fluid network development.

Several authors have also included a counterion pressure term, that is, in particular, dependent on the $\mathrm{pH}$ and other chemicals found in the solvent, in the free energy derivation presented above (Dobrynin and Rubinstein, 2005; Yashin and Balazs, 2006). However, we will not include this term in this paper for the following reasons. First, because the experiments of Boardman and Swartz (2003) are conducted in vivo in the tail tissue of the mouse we do not know what is the dominant chemical that triggers the onset of the lymphatic prepatterning. It could be any combination of the matrix metalloproteases and protons breaking down the cross-links between the fibres and the proton interaction with 
the fibres themselves. Therefore, we are including the simplest "illustrative" term that shows a potential mechanism of lymphatic network development in this manuscript. Secondly, the inclusion of the counterion pressure term (and indeed any other energy term that has been described in polymer gels) in the free energy does not actually change the mathematical detail of the analysis presented. Only the derivative of the free energy with respect to $\phi$ and $c$ at the steady state influences the linear stability and long wave analysis of the model presented below. Fundamentally, any mechanism that can contribute to gel swelling can also contribute to the patterning. However, we believe that the breaking down of the cross-links between the collagen fibers is the dominant mechanism. Finally, we feel that the inclusion of too many complicated free energy terms at this stage of the modeling of the lymphatic network development will lead to a false sense of security about the model. As pointed out above, there are several experimental issues that need to be addressed before the definitive model can be derived. In addition to this, we have not been able to find an experimental study that rigorously parameterizes all these free energy terms for collagen gels. Thus, for the purposes of transparency and simplicity, we will only consider the Flory-Huggins free energy terms as described above. We hope that this paper will encourage the experimental community to perform experiments on $2 \mathrm{D}$ collagen layers with varying either the matrix metallo protease concentrations or proton concentration.

Thus, we take the overall free energy of the two phase collagen mixture to be given by

$$
F=\int_{\Omega}\left[f(\phi, c)+\frac{k}{2}(\nabla \phi)^{2}\right] d V
$$

where

$$
f=\frac{k_{B} T}{v}\left\{(1-\phi) \ln (1-\phi)+\chi \phi(1-\phi)+\frac{1}{2 N_{x}}\left(\phi_{0}-\phi-\frac{1}{3} \phi \ln \frac{\phi_{0}}{\phi}\right)\right\} .
$$

In order to derive the equations describing the movement of collagen, we will use the variational principle that is used as standard in the polymer literature (Doi and Edwards, 1986; Doi, 1983; Tomari and Doi, 1995). It relies on minimizing the rate of energy dissipation, sometimes also called the Rayleighian. Development and more in depth discussion on this method can be found in Doi and Edwards (1986), Doi (1983). A good brief historical overview of this method is given in Hillert and Agren (2006) where it is also pointed out that unlike minimization of the Hamiltonian, which is a physical principle, minimizing the Rayleighian is more of a mathematical trick than a physical law.

Hence, after Doi and Onuki (1992), Hall et al. (2007), Milner (1993), Tanaka (1997), we need to minimize the following functional with respect to $\mathbf{v}_{f}$ and $\mathbf{v}_{p}$

$$
R=F_{t}+\int_{\Omega}\left\{\frac{1}{2} \xi\left(\mathbf{v}_{p}-\mathbf{v}_{f}\right)^{2}-p \nabla \cdot \mathbf{v}-\mathbf{v}_{p} \cdot \mathbf{F}_{p}-\mathbf{v}_{f} \cdot \mathbf{F}_{f}\right\} d V,
$$

where $\xi$ is the drag coefficient between the fibres and the fluid. Thus, the term $\frac{1}{2} \xi\left(\mathbf{v}_{p}-\mathbf{v}_{f}\right)^{2}$ represents the energy dissipation due to viscous forces in the two phase mixture. $\mathbf{F}_{i}$ are the forces acting on each of the components, and we have also introduced the pressure $p$ to guarantee the incompressibility condition $\nabla \cdot \mathbf{v}$. The variable $p$ is effectively a "Lagrange multiplier" that ensures the incompressibility of the fibre-fluid mixture. $F_{t}$ is the rate of change of free energy with time. 
The rate of change of free energy is derived by differentiating equation (11) with respect to time, i.e., we get

$$
F_{t}=\int_{\Omega}\left[\left(\frac{\partial f}{\partial \phi}-k \nabla^{2} \phi\right) \phi_{t}+\frac{\partial f}{\partial c} c_{t}\right] d V+k \int_{\partial \Omega} \phi_{t} \frac{\partial \phi}{\partial n} d S .
$$

From now on, we will define the natural boundary condition (Courant and Hilbert, 2004) originating from the correlation energy term to be

$$
\frac{\partial \phi}{\partial n}=0 \quad \text { on } \partial \Omega
$$

where $\frac{\partial}{\partial n}$ is the derivative normal to the domain boundary $\partial \Omega$, so that the second integral in (14) is zero.

From Eqs. (1) and (4), we can write $\phi_{t}=-\nabla \cdot\left(\phi \mathbf{v}_{p}\right)$ and $c_{t}=-\nabla \cdot\left(c(1-\phi) \mathbf{v}_{f}\right)+$ $\nabla \cdot(D(\phi) \nabla c)$ and substituting this into Eq. (14), we get

$$
\begin{aligned}
F_{t}= & -\int_{\Omega}\left\{\left(\frac{\partial f}{\partial \phi}-k \nabla^{2} \phi\right) \nabla \cdot\left(\phi \mathbf{v}_{p}\right)\right. \\
& \left.+\frac{\partial f}{\partial c}\left[\nabla \cdot\left(c(1-\phi) \mathbf{v}_{f}\right)-\nabla \cdot(D(\phi) \nabla c)\right]\right\} d V \\
= & \int_{\Omega}\left\{\phi \nabla\left(\frac{\partial f}{\partial \phi}-k \nabla^{2} \phi\right) \cdot \mathbf{v}_{p}+c(1-\phi) \nabla \frac{\partial f}{\partial c} \cdot \mathbf{v}_{f}+\frac{\partial f}{\partial c} \nabla \cdot(D(\phi) \nabla c)\right\} d V \\
& +\int_{\partial \Omega}\left[\left(\frac{\partial f}{\partial \phi}-k \nabla^{2} \phi\right) \phi \mathbf{v}_{p}+\frac{\partial f}{\partial c} c(1-\phi) \mathbf{v}_{f}\right] \cdot d \mathbf{S} .
\end{aligned}
$$

Assuming that there is no flux of fluid or collagen through the boundary (we will assume this throughout this paper), i.e., $\mathbf{v}_{f}$ and $\mathbf{v}_{p}$ are zero on $\partial \Omega$, we finally arrive at the following equation for the rate of change of free energy

$$
F_{t}=\left\{\phi \nabla\left(\frac{\partial f}{\partial \phi}-k \nabla^{2} \phi\right) \cdot \mathbf{v}_{p}+c(1-\phi) \nabla \frac{\partial f}{\partial c} \cdot \mathbf{v}_{f}+\frac{\partial f}{\partial c} \nabla \cdot(D(\phi) \nabla c)\right\} d V .
$$

Thus, by minimizing Eq. (13) with Eq. (17) with respect to $\mathbf{v}_{p}$ and $\mathbf{v}_{f}$, we get the following equations for $\mathbf{v}_{p}-\mathbf{v}_{f}$ and $\nabla p$

$$
\begin{aligned}
& -\xi\left(\mathbf{v}_{p}-\mathbf{v}_{f}\right)-\phi \nabla\left(\frac{\partial f}{\partial \phi}-k \nabla^{2} \phi\right)+\phi \nabla p+\mathbf{F}_{p}=0, \\
& \xi\left(\mathbf{v}_{p}-\mathbf{v}_{f}\right)-c(1-\phi) \nabla \frac{\partial f}{\partial c}+(1-\phi) \nabla p+\mathbf{F}_{f}=0 .
\end{aligned}
$$

From now on, we will assume that the forces $\mathbf{F}_{i}$ are zero, which essentially implies that we are assuming that the shear movement of the gel is small and all bulk movements are described by Flory mixing and elastic free energy terms in (11) and (12). We believe this to be realistic because there is no fluid or collagen flow across the implant and, therefore, the shear effects are likely to be small. 
Equations (1), (18), and (19) need either one more equation to tie them together since the Eqs. (18) and (19) are linearly dependent on the fluid and collagen velocity difference $\mathbf{v}_{p}-\mathbf{v}_{f}$. Alternatively, we would need to include higher order dissipative terms in $\mathbf{F}_{p}$ and $\mathbf{F}_{f}$. There are several ways of doing this. We will use the approach taken by Boissonade (2003), Wolgemuth et al. (2002) and introduce the so-called "bootstrap" equation that assumes that the phase averaged velocity is zero, i.e., we take everywhere

$$
\mathbf{v}=\phi \mathbf{v}_{p}+(1-\phi) \mathbf{v}_{f}=0 \text {. }
$$

The "bootstrap expression" given by Eq. (20) is clearly valid when there is no flux of collagen or fluid through the boundaries. In this paper, we will limit ourselves to this case only, but in future publications, we will address the case when there are sources/sinks at the boundary.

Thus, combining Eqs. (18)-(20), we get

$$
\begin{aligned}
& \xi \mathbf{v}_{p}=-\phi(1-\phi)^{2}\left[\left(f_{\phi \phi}-c f_{\phi c}\right) \nabla \phi+\left(f_{\phi c}-c f_{c c}\right) \nabla c-k \nabla \nabla^{2} \phi\right], \\
& \xi \mathbf{v}_{f}=\phi^{2}(1-\phi)\left[\left(f_{\phi \phi}-c f_{\phi c}\right) \nabla \phi+\left(f_{\phi c}-c f_{c c}\right) \nabla c-k \nabla \nabla^{2} \phi\right],
\end{aligned}
$$

where $f_{\phi \phi}=\frac{\partial^{2} f}{\partial \phi^{2}}, f_{\phi c}=\frac{\partial^{2} f}{\partial \phi \partial c}, f_{c c}=\frac{\partial^{2} f}{\partial c^{2}}$.

The representative set of parameter values is shown in Table 1.

Substituting Eqs. (21) and (22) into conservation Eqs. (1) and (4), we get two coupled equations for $\phi$ and $c$. After having nondimensionalized these by scaling $\mathbf{x} \sim$ $L=2 \times 10^{-3} \mathrm{~m}$ (representative size of the sample in Boardman and Swartz, 2003), $\mathbf{v}_{p}, \mathbf{v}_{f} \sim[v]=k_{b} T /(\nu \xi L)=10^{-8} \mathrm{~ms}^{-1}, c \sim c_{\infty}$ and $t \sim L /[v]$ we get the following dimensionless equations for $\phi$ and $c$

$$
\begin{aligned}
\phi_{t}= & \nabla \cdot\left\{\phi^{2}(1-\phi)^{2}\left[\left(f_{\phi \phi}-c f_{\phi c}\right) \nabla \phi-\kappa \nabla \cdot\left(\nabla^{2} \phi\right)+\left(f_{\phi c}-c f_{c c}\right) \nabla c\right]\right\}, \\
c_{t}= & \nabla \cdot\left\{-c \phi^{2}(1-\phi)^{2}\left[\left(f_{\phi \phi}-c f_{\phi c}\right) \nabla \phi-\kappa \nabla \cdot\left(\nabla^{2} \phi\right)+\left(f_{\phi c}-c f_{c c}\right) \nabla c\right]\right\} \\
& +(1 / P e) \nabla \cdot\{D(\phi) \nabla c\},
\end{aligned}
$$

where $\kappa=\frac{v k}{k_{B} T L^{2}}, P e=\frac{D_{0}}{L[v]}, N_{x}=N_{x 0} e^{\alpha \frac{c-c 0}{1-c}}, D(\phi)=e^{-a\left(\phi^{b}-\phi_{0}^{b}\right)}$, and $c_{0} \rightarrow c_{0} / c_{\infty}$, and

$$
f=(1-\phi) \ln (1-\phi)+\chi \phi(1-\phi)+\frac{1}{2 N_{x}}\left(\phi_{0}-\phi-\frac{1}{3} \phi \ln \frac{\phi_{0}}{\phi}\right) .
$$

From now on, we will use the following dimensionless parameter values (see also Table 1): $\phi_{0}=0.03, \chi=0.5, c_{0}=0.1, P e=0.05, N_{x 0}=10, \kappa=0.1$, and $\alpha=250$.

We will solve the Eqs. (23) and (24) for two cases. First, we solve it in one dimension with periodic boundary conditions. Secondly, in two dimensions in the rectangle, we will use periodic boundary conditions in the $y$ direction to reflect the fact that the sample is circular around the mouse tail. In the other, $x$ direction, we use a zero flux of collagen boundary condition in addition to the natural boundary condition $\frac{\partial \phi}{\partial n}=0$, which originated from the correlation energy term in Eqs. (14) and (15). As boundary conditions for $c$ in the $x$ direction, we use permeability boundary conditions at the inlet and outlet to reflect 
Table 1 Summary of dimensional parameters used as input to the model along with their source/reference

\begin{tabular}{|c|c|c|c|c|}
\hline Parameter & Value & Units & Description & Reference \\
\hline$k_{B} T / v$ & $4.1 \times 10^{3}$ & $\mathrm{~Pa}$ & $\begin{array}{l}\text { thermal energy per } \\
\text { volume of monomer }\end{array}$ & Wolgemuth et al. (2004) \\
\hline$\xi$ & $2 \times 10^{14}$ & $\mathrm{~N} \mathrm{~s} \mathrm{~m}^{-4}$ & $\begin{array}{l}\text { drag coefficient between } \\
\text { fibres and fluid }\end{array}$ & Wolgemuth et al. (2004) \\
\hline$\chi$ & 0.5 & & $\begin{array}{l}\text { Flory interaction } \\
\text { parameter }\end{array}$ & Wolgemuth et al. (2004) \\
\hline$D_{0}$ & $10^{-10}$ to $10^{-8}$ & $\mathrm{~m}^{2} \mathrm{~s}^{-1}$ & $\begin{array}{l}\text { Diffusivity of molecules } \\
\text { and protons }\end{array}$ & $\begin{array}{l}\text { Perry and Green (1997), } \\
\text { Agmon (1995), Tuckerman } \\
\text { et al. (2006) }\end{array}$ \\
\hline$k$ & $1.6 \times 10^{-3}$ & $\mathrm{~N}$ & $\begin{array}{l}\text { Correlation energy } \\
\text { parameters }\end{array}$ & Wolgemuth et al. (2004) ${ }^{\mathrm{a}}$ \\
\hline$\phi_{0}$ & 0.03 & & $\begin{array}{l}\text { Initial collagen volume } \\
\text { fraction }\end{array}$ & Boardman and Swartz (2003) \\
\hline$c_{\infty}$ & $10^{8}$ & M & $\begin{array}{l}\text { Value of solute } \\
\text { concentration when all the } \\
\text { cross-links have broken }\end{array}$ & Chosen \\
\hline
\end{tabular}

${ }^{\mathrm{a}}$ Chosen so that the correlation occurs over lengthscale of $\mathrm{mm}$

the fact that the $\mathrm{pH}\left(c_{0}\right)$ and permeability of the surrounding tissue/media can be very different from the $\mathrm{pH}$ of the collagen implant, i.e., we choose

$$
\begin{aligned}
& -\mathbf{n} \cdot \frac{1}{P e} D(\phi) \nabla c=\beta\left(c_{0}-c\right) \quad \text { at } x=0 \text { and } x=L, \\
& \mathbf{n} \cdot \nabla \phi=0, \quad \text { and } \quad \mathbf{n} \cdot \mathbf{v}_{p}=0, \quad x=0 \text { and } x=L .
\end{aligned}
$$

\section{Linear stability analysis}

The aim of this section is to investigate if the uniform steady state, given by $\phi_{0}$ and $c_{0}$, is linearly stable to small spatially varying perturbations. Thus, we begin by looking for a solution to linearized equations (23) and (24) around the uniform steady state $\phi_{0}$ and $c_{0}$ in the form $\propto e^{\sigma t+i \mathbf{k} \cdot \mathbf{x}}$. This gives us the dispersion relation

$$
\begin{gathered}
\sigma^{2}+\sigma k^{2} \phi_{0}^{2}\left(1-\phi_{0}\right)^{2}\left[f_{\phi \phi}-2 c_{0} f_{\phi c}+\frac{1}{P e \phi_{0}^{2}\left(1-\phi_{0}\right)^{2}}+\kappa k^{2}\right] \\
+k^{4} \frac{\phi_{0}^{2}\left(1-\phi_{0}\right)^{2}}{P e}\left(f_{\phi \phi}-c_{0} f_{\phi c}+\kappa k^{2}\right)=0
\end{gathered}
$$

where $f_{\phi \phi}=\left.\frac{\partial^{2} f}{\partial \phi^{2}}\right|_{\left(\phi_{0}, c_{0}\right)}$ and $f_{\phi c}=\left.\frac{\partial^{2} f}{\partial \phi \partial c}\right|_{\left(\phi_{0}, c_{0}\right)}$, and $f$ is given by Eq. (25).

The steady state is stable if both coefficients in the above polynomial are positive and this gives us the following conditions of stability

$$
f_{\phi \phi}-c_{0} f_{\phi c}+\kappa k^{2}>0, \quad \text { and } \quad \frac{1}{P e \phi_{0}^{2}\left(1-\phi_{0}\right)^{2}}-c_{0} f_{\phi c}>0
$$




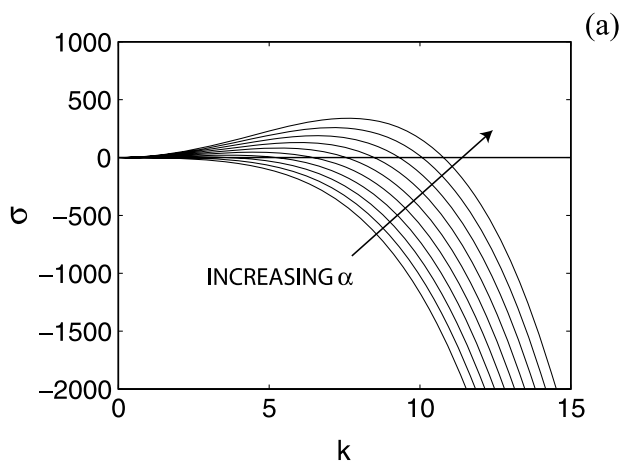

(a)

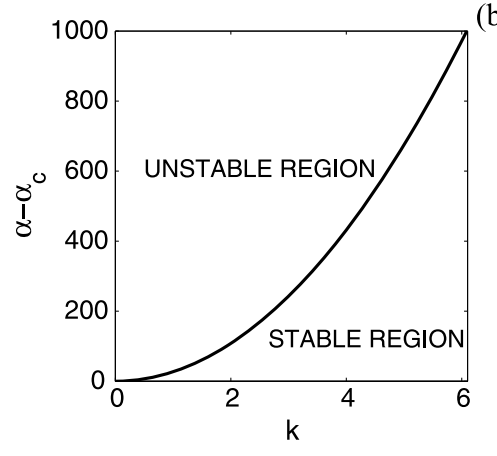

(c)

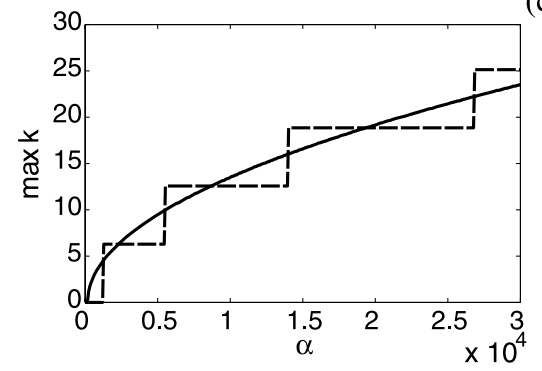

(b)

Fig. 2 (a): Dispersion relation for $\alpha$ varying from 0 to 4,000 with step interval of 400 between the graphs shown. (b): Neutral stability curve; $\alpha_{c}=458.350$. (c): Fastest growing modes in the case of infinite domain (solid line) and in the case of finite domain $x=[0,1]$ (dashed line).

The shape of the dispersion relation is shown on Fig. 2a for different values of the parameter $\alpha$ for fixed $c_{0}=0.1$ and on Fig. 3a for different values of $c_{0}$ with fixed $\alpha=250$.

From (29), we see that a spatial pattern forming instability can occur for wave numbers $0<k<k_{c}=(1 / \kappa)\left(c_{0} f_{\phi c}-f_{\phi \phi}\right)$ provided that $c_{0} f_{\phi c}-f_{\phi \phi}>0$ and that the second of the stability conditions (29) is always satisfied. In this case, $k=0$ and $k=k_{c}$ are neutrally stable. The fastest growing mode as a function of $\alpha$ for a fixed $c_{0}=0.1$ is shown on Fig. 2c and as a function of $c_{0}$ for a fixed value of $\alpha=250$ is shown on Fig. 3c. The neutral stability curve is given ${ }^{2}$ by

$$
N\left(k ; c_{0}, \alpha\right)=\left(d_{11}+\kappa k^{2}\right) k^{4}=0,
$$

where

$$
d_{11}=f_{\phi \phi}-c_{0} f_{\phi c}=\frac{1}{1-\phi_{0}}-2 \chi+\frac{1}{2 \phi_{0} N_{x 0}}-\alpha \frac{c_{0}}{2 N_{x 0}\left(1-c_{0}\right)} .
$$

The neutral stability curves for two different cases of bifurcation parameter ( $\alpha$ and $\left.c_{0}\right)$ are shown on Figs. $2 b$ and $3 b$.

\footnotetext{
${ }^{2}$ We will consider two bifurcation parameters for our analysis, $\alpha$ and $c_{0}$.
} 


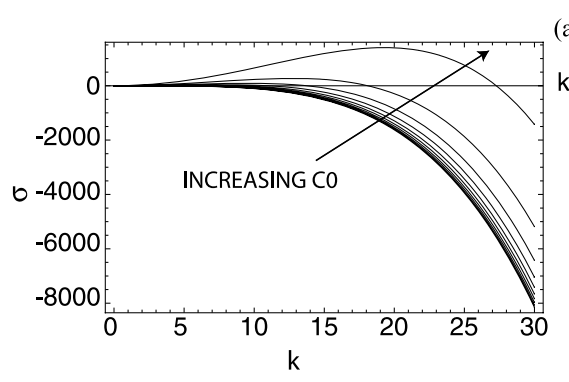

50

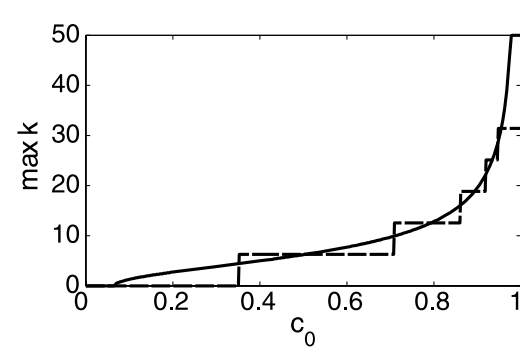

(b)

Fig. 3 (a): Dispersion relation for $c$ varying from 0 to 0.9 with step interval of 0.1 between the graphs shown. (b): Neutral stability curve; $c_{c r i t}=0.0657506$. (c): Fastest growing modes in the case of infinite domain (solid line) and in the case of finite domain $x=[0,1]$ (dashed line).
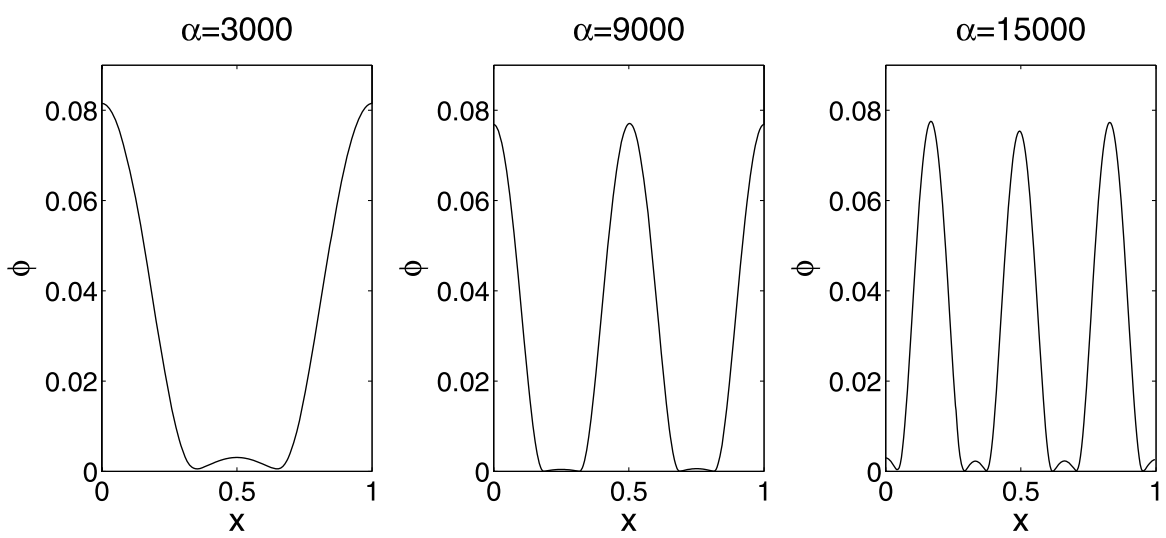

Fig. 4 Numerical solutions for different $\alpha \mathrm{s}$ at $t=100$ obtained using Comsol Multiphysics finite element package.

Numerical solutions of the full nonlinear model given by (23) and (24) at different values of $\alpha$ for fixed $c_{0}$ are shown on Fig. 4. The solutions for fixed $\alpha$ and varying $c_{0}$ as a bifurcation parameter, for values that correspond to period one, two and three solutions, are virtually indistinguishable from these and we do not show them here.

We can see by comparing the numerical solutions shown on Fig. 4 with the analytic predictions of the fastest growing mode, shown on Fig. $2 \mathrm{c}$ that they agree very well. Therefore, we can conclude that the analytic estimate for the fastest growing mode that 


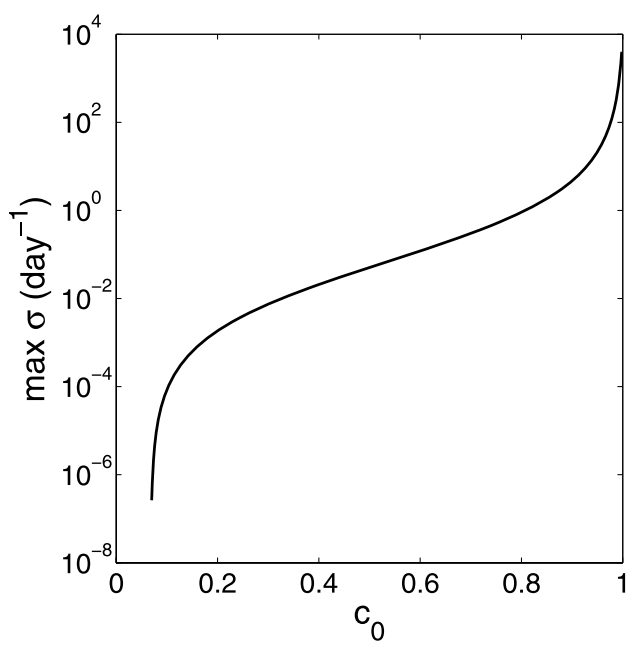

Fig. 5 Maximum value of $\sigma$ as a function of the bifurcation parameter $c_{0}$. Corresponding values for maximum $k$ are shown on Fig. 3c.
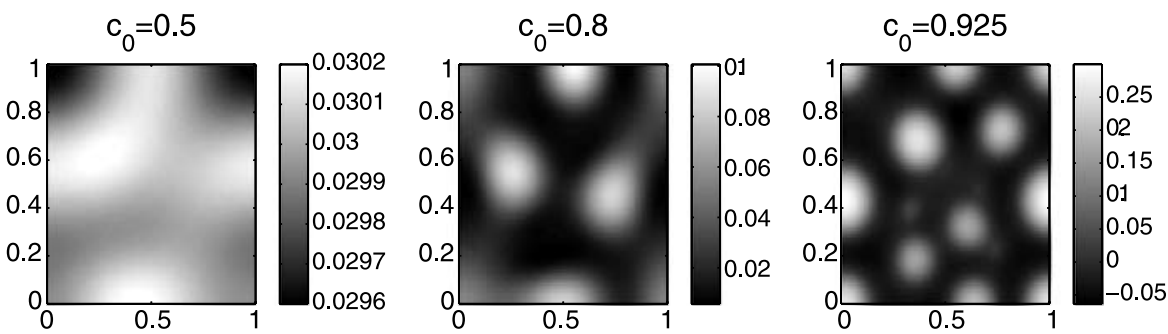

Fig. 6 Results of numerical simulation for three different cases of bifurcation parameter $c_{0}=0.5,0.8$, and 0.925 , corresponding to period 1,2 , and 3, respectively, according to linear stability analysis. Results are shown at dimensionless time $t=2$ which corresponds to dimensional time of about 6 days.

we found using linear stability theory is indeed consistent with numerics. On Fig. 5, we show the dimensional values for the maximum growth rate as a function of the bifurcation variable $c_{0}$. In the experiments of Boardman and Swartz (2003), the patterns are seen to be starting to be observed after about 10 days, which seems to agree with our estimates for the maximum growth rate of order $0.1-0.01 \mathrm{day}^{-1}$ for range $c_{0}=0.6-0.8$. But, of course, we need to stress here that because these experiments are conducted in vivo, there are other factors, such as lymphatic endothelial cells and epidermal cells migrating into the implant, in play and the direct comparison with the experiments is not possible. Instead, we can only say that our predicted timescales for patterning are not contradicted by the experimental findings.

On Fig. 6, we show the two dimensional simulations for three different values of bifurcation parameter $c_{0}$ that according to linear stability analysis correspond to periods 1,2 , and 3 patterns. As one can see the linear stability analysis is consistent with the numerical simulations about the fastest growing point. We would also like to point out that period 3 
simulations are already starting to show geometric organization of pattern into a hexagonal looking pattern (top left-hand side panel for case $c_{0}=0.925$ ). We will present a more detailed nonlinear analysis for hexagonal lattice solutions that takes into account higher order terms in expansion, together with more detailed two dimensional simulations in a separate publication.

\section{Long wavelength reduction}

We notice that at the bifurcation point $c=c_{c r i t}$, there is a double zero of a dispersion relation at zero wave number. Therefore, a small amount above the bifurcation point the modes that start growing are the ones with long wavelength. This suggests that we might be able to find simplified equations using the long wavelength reduction (Kozyreff and Tlidi, 2004). We do this by scaling space and time suitably and then expanding the state variables as series in the small parameter. We will discuss the choice of the small parameter, scalings, and expansion in detail now.

The natural small parameter to use in our current problem is $\kappa=0.1$, i.e., the parameter that quantifies over which length scale we might expect sharp variations in collagen volume fraction. Thus, we take $\varepsilon=\kappa=0.1$. The scalings suitable for time and space can be deduced by considering the neutral stability curve near the bifurcation point, i.e.,

$$
\frac{d N}{d k}=6 \varepsilon k^{3}\left(\frac{2 d_{11}}{3 \varepsilon}+k^{2}\right),
$$

which suggest that if the bifurcation parameter is above the bifurcation point by the value that makes $d_{11} \sim \varepsilon^{2}$, then to balance the terms in the brackets we need $k \sim \sqrt{\varepsilon}$. With this choice of scaling, the dispersion relation suggests that $\sigma \sim \varepsilon^{3}$ for all terms to balance.

These scalings derived from linear stability analysis for $\sigma$ and $k$ translate into scalings of time $T=\varepsilon^{3} t$ and space $\mathbf{X}=\sqrt{\varepsilon} \mathbf{x}$. This transforms our equations to

$$
\begin{aligned}
& \varepsilon^{2} \phi_{T}=\nabla \cdot\left\{\phi^{2}(1-\phi)^{2}\left[\varepsilon^{2} d_{11}^{*} \nabla \phi+d_{12} \nabla c-\varepsilon^{2} \nabla \nabla^{2} \phi\right]\right\}, \\
& \varepsilon^{2} c_{T}=\nabla \cdot\left\{-c \phi^{2}(1-\phi)^{2}\left[\varepsilon^{2} d_{11}^{*} \nabla \phi+d_{12} \nabla c-\varepsilon^{2} \nabla \nabla^{2} \phi\right]+\frac{1}{P e} D \nabla c\right\},
\end{aligned}
$$

where $d_{11}=\varepsilon^{2} d_{11}^{*}$ (from now on we will drop $*$ ).

The linear stability of Eq. (23) suggest the scalings to $\phi$ and $c$. Since the time derivative term and derivatives of $\phi$ with the scalings presented above are $O\left(\varepsilon^{3}\right)$ and the derivatives in $c$ are $O(\varepsilon)$, we expect that the perturbations in $\phi \sim 1$ and $c \sim \varepsilon^{2}$. Thus, we expand $\phi$ and $c$ in the following asymptotic series

$$
\begin{aligned}
& \phi(\mathbf{X}, T)=\phi_{00}(\mathbf{X}, T)+\varepsilon \phi_{1}(\mathbf{X}, T)+\cdots, \\
& c(\mathbf{X}, T)=c_{00}+\varepsilon^{2} c_{2}(\mathbf{X}, T)+\cdots
\end{aligned}
$$

We are seeking for a solution to the Eqs. (33) and (34) in two dimensions on the rectangle with periodic boundary conditions in $y$ direction and zero flux of polymer across the boundaries in $x$ direction. The boundary condition on $c$ in the $x$ direction reflects the 
fact that protons can permeate through from the edges of the sample and that this rate is proportional to the permeability $\beta$. Thus, the boundary conditions in the $x$ direction become

$$
\phi_{x}=0, \quad d_{12} c_{x}-\varepsilon^{2}\left(\nabla^{2} \phi\right)_{x}=0, \quad \frac{1}{P e} D c_{x}=\beta\left(c_{0}-c\right),
$$

after long wavelength scaling.

Using the expansions (35) and (36), we find that at $O(1) c_{00}=c_{0}=$ constant everywhere.

At order $O\left(\varepsilon^{2}\right)$, Eq. (34) gives

$$
\begin{aligned}
0= & \nabla \cdot\left\{-c_{0} \phi_{00}^{2}\left(1-\phi_{00}\right)^{2}\left[d_{11} \nabla \phi_{00}-\nabla \nabla^{2} \phi_{00}\right]\right\} \\
& +\nabla \cdot\left\{\left[\frac{1}{P e} D\left(\phi_{00}\right)-c_{0} \phi_{00}^{2}\left(1-\phi_{00}\right)^{2} d_{12}\right] \nabla c_{2}\right\} .
\end{aligned}
$$

Solving this equation gives us

$$
\begin{aligned}
& -c_{0} \phi_{00}^{2}\left(1-\phi_{00}\right)^{2}\left[d_{11} \nabla \phi_{00}-\nabla \nabla^{2} \phi_{00}\right] \\
& \quad+\left[\frac{1}{P e} D\left(\phi_{00}\right)-c_{0} \phi_{00}^{2}\left(1-\phi_{00}\right)^{2} d_{12}\right] \nabla c_{2}=\nabla \times \mathbf{F},
\end{aligned}
$$

where $\mathbf{F}$ is an arbitrary vector field that will be determined using the boundary conditions. When the permeability of the boundary to proton ions is much smaller than the diffusion of protons within the sample, i.e., $\beta P e \sim O(\varepsilon)$, then the boundary condition for $c$ at $O\left(\varepsilon^{2}\right)$ becomes $c_{2, x}=0$ at the boundary. Hence, the component normal to the boundary at $x=0$ and $x=1$ of the left-hand side of (39) is zero and this means that in two dimensions $x$ and $y$ components of $\nabla \times \mathbf{F}$ are both zero and, therefore, we can write for our two dimensional problem that

$$
\nabla c_{2}=\frac{c_{0} \phi_{00}^{2}\left(1-\phi_{00}\right)^{2}}{\frac{1}{P e} D\left(\phi_{00}\right)-c_{0} \phi_{00}^{2}\left(1-\phi_{00}\right)^{2} d_{12}}\left[d_{11} \nabla \phi_{00}-\nabla \nabla^{2} \phi_{00}\right] .
$$

Thus, the $O\left(\varepsilon^{2}\right)$ equation for $\phi_{00}$ is now given by (after dropping subindexes 00 )

$$
\phi_{T}=\nabla \cdot\left\{g(\phi)\left[d_{11} \nabla \phi-\nabla \nabla^{2} \phi\right)\right\},
$$

where

$$
\begin{aligned}
& g(\phi)=\phi^{2}(1-\phi)^{2} \frac{D(\phi) / P e}{D(\phi) / P e-c_{0} \phi^{2}(1-\phi)^{2} d_{12}}, \\
& d_{11}=f_{\phi \phi}-c_{0} f_{\phi c}, \quad d_{12}=f_{\phi c}-c_{0} f_{c c},
\end{aligned}
$$

are all evaluated at $\phi$. This equation is well behaved and regular only if $g$ does not have any singularities. This highlights the fact that we need $D(\phi) / P e-c_{0} \phi^{2}(1-\phi)^{2} d_{12}>0$, i.e., the proton concentration is always moving from higher to lower and not vice versa. In 


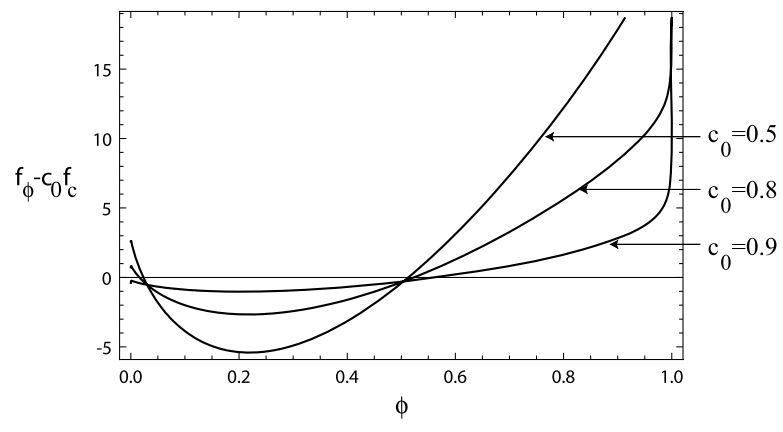

Fig. 7 Profiles of $f_{\phi}-c_{0} f_{c}$ in Eq. (45) for different values of $c_{0}$.

other words, we will not allow protons to accumulate due to collagen movement. Having said that, when $P e \ll 1$, as in our case, we can simplify the expression for $g(\phi)$. We obtain

$$
g(\phi) \approx \phi^{2}(1-\phi)^{2}
$$

provided $P e c_{0} \phi^{2}(1-\phi)^{2} d_{12} \ll 1$.

Thus, the long wavelength reduction results in a nonlinear Cahn-Hilliard equation with boundary conditions $\phi_{x}=0$ and $\left(\nabla^{2} \phi\right)_{x}=0$ at the boundaries in the $x$ direction and periodic in the $y$ direction.

We could rewrite the Eqs. (41) in an alternative form

$$
\phi_{T}=\nabla \cdot\left\{g(\phi) \nabla\left[f_{\phi}-c_{0} f_{c}-\nabla^{2} \phi\right]\right\} .
$$

Translating the equation back into the unscaled coordinates used in previous section, we get the equation

$$
\phi_{t}=\nabla \cdot\left\{g(\phi) \nabla\left(h(\phi)-\epsilon \nabla^{2} \phi\right)\right\},
$$

where $g(\phi)$ is given by Eq. (42), and $h(\phi)$ is given by

$$
\begin{aligned}
h(\phi)= & -1+(1-2 \phi) \chi-\log (1-\phi)-\frac{1}{6 N_{x 0}}\left(2+\log \frac{\phi_{0}}{\phi}\right) \\
& +\frac{\alpha c_{0}}{2 N_{x 0}\left(1-c_{0}\right)}\left(\phi_{0}-\phi-\frac{1}{3} \phi \log \frac{\phi_{0}}{\phi}\right) .
\end{aligned}
$$

Profiles of $h(\phi)$ are shown on Fig. 7.

The numerical solution computed using the reduced Eq. (41) is shown on Fig. 8

As we can see the pattern emerges over the dimensionless timescale of order $t \sim 15$ which translates into dimensional timescale of order 30 days. This finding is consistent with the experimental observations of Boardman and Swartz (2003). On the domain shown on Fig. 8, the linear stability analysis predicted that the fastest solution should be period 6 solution and this is indeed consistent with the numerical results. 

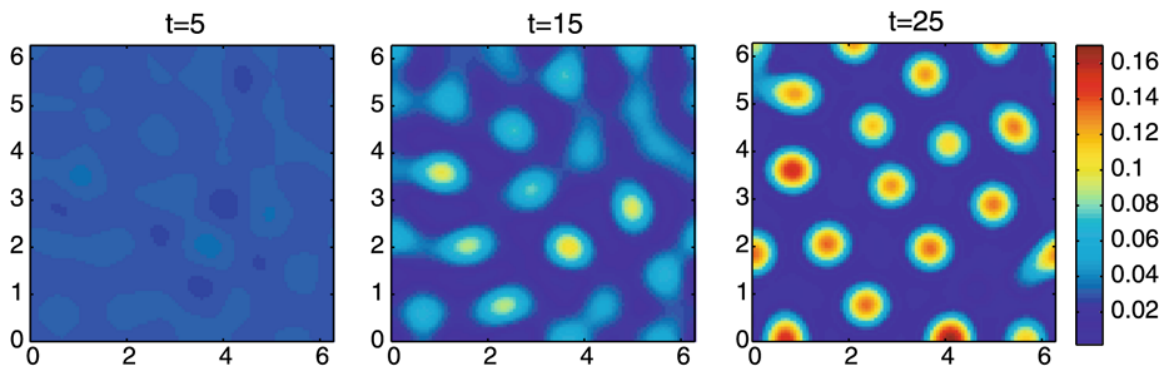

Fig. 8 Solutions of Eq. (41) for parameter values $c_{0}=0.9, \alpha=240, \chi=0.5, N_{x, 0}=10$, and $\phi_{0}=0.03$ at different time points. Initial condition was taken to be a small random perturbation around the steady state $\phi_{0}$.

\section{Discussion}

In this paper, we have presented a model that for the first time, quantitatively describes the factors that influence the first stage of lymphatic network formation within biogels. We show that due to the fact that solutes, such as protons, can break down the crosslinks between collagen fibres, therefore, decreasing the elastic constraining forces on the collagen gel (i.e., Flory elastic energy), an initially homogeneous collagen gel is unstable to small spatial perturbations if the solute concentration is higher than a critical level determined by the linear stability analysis presented in Section 3.

We have also found that while the linear stability analysis predicts the wave number that will grow fastest, it also predicts that the zero wave number is always neutrally stable and that the bifurcation from stable to unstable regime occurs there. This means that slightly above the bifurcation point the fastest growing mode has a long wavelength. This inspired us to use long wavelength reduction reminiscent of that used as standard in fluid dynamics and optics, and it enabled us to simplify coupled fourth order partial differential equations and derive a single equation for a collagen gel volume fraction that is very similar to the Cahn-Hilliard equation. We found using numerical simulations that the system appears to be moving toward a hexagonal looking pattern, which is very similar to the real lymphatic network that develops in experiments of Boardman and Swartz (2003). We will present a nonlinear analysis of the stability and evolution of the hexagonal patterns in a subsequent publication.

Of course, in the end, lymphatics are not just patterns in the collagen gel, but are formed by lymphatic endothelial cells in cellular media. Our theory, therefore, helps to explain the different cascade of events that would lead to it. We believe that collagen arranges into a prepattern and then lymphatic endothelial cells migrate into the gel to make lymphatic vessels. This is realistic assuming that lymphatic endothelial cells have similar behavior to vascular endothelial cells, i.e., that they migrate fastest at a finite, nonzero collagen volume fraction (Zaman et al., 2005). There are no measurements similar to Wolf et al. (2003), Zaman et al. $(2005,2006)$ that show that this would be true for lymphatic endothelial cells. However, it is intuitively clear that if it is true for endothelial cells and cancer cells then this could also be true for lymphatic endothelial cells. Thus, following the prepatterning of the collagen gel, the lymphatic endothelial cells have to invade the gel in the fastest possible way to form the hexagonal looking lymphatic structures without needing any extra complex cell signaling to tell them anything about the morphology. 


\section{Acknowledgements}

TR would like to acknowledge the support of the Royal Society by its award of the University Research Fellowship and for the help received from Drs. David Smith, Marvin Jones, and Gregory Kozyreff when preparing this manuscript. ACF acknowledges the support of the Mathematics Applications Consortium for Science and Industry (www.macsi.ul.ie) funded by the Science Foundation Ireland mathematics initiative grant 06/MI/005.

\section{References}

Agmon, N., 1995. The grotthouse mechanism. Chem. Phys. Lett. 244, 456-462.

Boardman, K.C., Swartz, M.A., 2003. Intersititial flow as a guide for lymphatics. Circ. Res. 92, 801-808.

Boissonade, J., 2003. Simple chemomechanical process for self-generation of rhythms and forms. Phys. Rev. Lett. 90, 1-4.

Boudaoud, A., Caieb, S., 2003. Mechanical phase diagram of shrinking cylindrical gels. Phys. Rev. E 68, 021801-1-6.

Cassella, M., Skobe, M., 2002. Lymphatic vessel activation in cancer. Ann. N.Y. Acad. Sci. 979, 120-130.

Clague, D.S., Phillips, R.J., 1997. A numerical calculation of the hydraulic permeability of threedimensional disordered fibrous media. Phys. Fluids 9, 1562-1572.

Cogan, N.G., Keener, J.P., 2005. Channel formation in gels. SIAM J. Appl. Math. 65, 1839-1854.

Courant, R., Hilbert, D., 2004. Methods of Mathematical Physics, vol. 1. Wiley-VCH Verlag GmbH and CO. KGaA.

Dobrynin, A.V., Rubinstein, M., 2005. Theory of polyelectrolytes in solutions and at surfaces. Prog. Polym. Sci. 30, 1049-1118.

Doi, F., Edwards, S.F., 1986. The Theory of Polymer Dynamics. Oxford University Press, Oxford.

Doi, M., 1983. Variational principle for the Kirkwood theory for the dynamics of polymer solutions and suspensions. J. Chem. Phys. 79, 5080-5087.

Doi, M., Onuki, A., 1992. Dynamic coupling between stress and composition in polymer solutions and blends. J. Phys. II France 2, 1631-1656.

Flory, J.P., 1953. Principles of Polymer Chemistry. Cornell University Press, Ithaca.

Hall, D.M., Lookman, T., Fredrickson, G.H., Banerjee, S., 2007. Numerical method for hydrodynamic transport of inhomogeneous polymer melts. J. Comput. Phys 224, 681-698.

Hillert, M., Agren, J., 2006. Extremum principles for irrevercable processes. Acta Mater. 54, 2063-2066.

Jain, R.K., 2001. Delivery of molecular and cellular medicine to solid tumors. Adv. Drug Deliv. Rev. 46, 149-168.

Jussila, L., Alitalo, K., 2002. Vascular growth factors and lymphangiogenesis. Physiol. Rev. 82, 673-700.

Kozyreff, G., Tlidi, M., 2004. Optical patterns with different wavelengths. Phys. Rev. E 69, 066202-1-11.

Landis, E.M., Pappenheimer, J.R., 1963. Exchange of Substances through the Capillary Walls, Handbook of Physiology: Circulation II, pp. 961-1034.

Levick, J.R., 1987. Flow through interstitium and other fibrous matrices. Q. J. Exp. Physiol. 72, 409-438.

Milner, S.T., 1993. Dynamical theory of concentration fluctuations in polymer solutions under shear. Phys. Rev. E 48, 3674-3691.

Nussbaum, J.H., 1986. Electric Field Control of Mechanical and Electrochemical Properties of Polyelectrolyte Gel Membranes, PhD thesis, Massachusetts Institute of Technology.

Nussbaum, J.H., Grodzinsky, A.J., 1981. Proton diffusion reaction in a protein polyelectrolyte membrane and the kinetics of electromechanical forces. J. Membr. Sci. 8, 193-219.

Perry, R.H., Green, D.W., 1997. Perry's Chemical Engineers' Handbook, 7th ed. McGraw-Hill, New York.

Skobe, M., Hawighorst, T., Jackson, D.G., Prevo, R., Janes, L., Velasco, P., Riccardi, L., Alitalo, K., Claffey, K., Detmar, M., 2001. Induction of tumor lymphangiogenesis by VEGF-C promotes breast cancer metastasis. Nat. Med. 7, 192-198.

Stacker, S.A., Achen, M.G., Jussila, L., Baldwin, M.E., Alitalo, K., 2002. Lymphangiogenesis and cancer metastasis. Nat. Rev. Cancer 2, 573-583.

Tanaka, H., 1997. Viscoelastic model of phase separation. Phys. Rev. E 56, 4451-4462.

Tomari, T., Doi, M., 1995. Hysteresis and incubation in the dynamics of volume transition of spherical gels. Macromolecules 28, 8334-8343. 
Tuckerman, M.E., Chandra, A., Marx, D., 2006. Structure and dynamics of $\mathrm{OH}^{-}$(aq). Acc. Chem. Res. 39, 151-158.

Vogel, S., 2003. Comparative Biomechanics, Life's Physical World. Princeton University Press, Princeton.

Watson, P.D., Bell, D.R., Renkin, E.M., 1980. Early kinetics of large molecule transport between plasma and lymph in dogs. Am. J. Physiol. 239, H525-H531.

Watson, P.D., Grodins, F.S., 1978. An analysis of the effects of the interstitial matrix on plasma-lymph transport. Microvasc. Res. 16, 19-41.

Wolf, K., Mazo, I., Leung, H., Engelke, K., von Andrian, U.H., Deryugina, E.I., Strongin, A.Y., Broker, E.-B., Friedl, P., 2003. Compensation mechanism in tumour cell migration: mesenchymal-amoeboid transition after blocking of pericellular proteolysis. J. Cell Biol. 160, 267-277.

Wolgemuth, C., Hoiczyk, E., Kaiser, D., Oster, G., 2002. How myxobacteria glide. Curr. Biol. 12, 369377.

Wolgemuth, C.W., Mogilner, A., Oster, G., 2004. The hydration dynamics of polyelectrolyte gels with applications to cell motility and drug delivery. Eur. Biophys. J. 33, 146-158.

Yashin, V.V., Balazs, A.C., 2006. Pattern formation and shape changes in self-oscillating polymer gels. Science 314, 798-801.

Zaman, M.H., Kamm, R.D., Patsudaira, P., Lauffenburger, D.A., 2005. Computational model for cell migration in three-dimensional matrices. Biophys. J. 89, 1389-1397.

Zaman, M.H., Trapani, L.M., Siemeski, A., MacKellar, D., Gong, H., Kamm, R.D., Wells, A., Lauffenburger, D.A., Matsudaira, P., 2006. Migration of tumor cells in 3d matrices is governed by matrix stiffness along with cell-matrix adhesion and proteolysis. Proc. Natl. Acad. Sci. 103, 10889-10894. 